\title{
Feasibility of a set of wrist-worn novice devices for dual motion comparison of the upper limbs during lateral raise motions
}

\author{
Haemi Jee* \\ Department of Physical Therapy, Namseoul University, Cheonan, Korea
}

Comparison of the upper limbs during natural kinematic motions is necessary for early detection of musculoskeletal imbalance between ipsilateral and contralateral sides in natural settings. Therefore, this study aims to evaluate the feasibility of a novice set of wrist-worn devices designed to assess and compare the dual kinematic motions of the upper limbs during lateral raises. The test-retest and the golden standard and novice device result comparisons were conducted for feasibility assessment of the novice set of devices. Pearson correlation coefficients between 0.65 and $0.88(P<0.01)$ and effect sizes between 0.02 and 0.42 indicated feasible application of the novice devices. Considering correlation coefficient of 0.65 between the left and right upper limbs, the results show applicable feasibility of the novice device during lateral raises. In conclusion, the novice set of devices for comparing dual upper limb motions may be applied to assessing and comparing dual upper limb motions for limb motion comparisons and early detection of dysfunctional movements between the limbs.

Keywords: Asymmetry, Kinematics, Bilateral motion, Dyskinesia

\section{INTRODUCTION}

Identification of abnormal movements during exercise or everyday activities may allow detection of further dealignment of the kinematic chain of the body for possible progression into musculoskeletal disorder (do Rosário, 2014; Hadzic et al., 2014; Maly et al., 2015). Despite the importance of early and precise detection of dysfunction in early stage, observing the difference is not an easy task and scientifically inaccurate (do Rosário, 2014). Various assessment systems or devices such as three-dimensional (3D) motion analysis system, inertial sensors, optical marker tracking system, photography analysis, or force platforms have been developed and utilized to assess the kinematics of human motions for the purpose of athletic training and musculoskeletal disorder (do Rosário, 2014; Matsuki et al., 2011; Rettig et al., 2015). In addition to such systems or devices, smartphone-based movement detection systems have been gaining significant attention in recent years with the global-wide IoT (Internet of Things) frenzy.
Despite the wide-spread attention and numerous novice systems and devices that have been introduced in recent years, there have been reports of different problems. Assessment inaccuracy, limitation of assessment, and assessment limitation have been reported as some of the problems (do Rosário, 2014; Matsuki et al., 2011). For example, although analyzing photographed pictures may provide stable analytic results, since pictures provide two-dimensional information, certain movements may provide limited information at certain angles for accurate assessment. Three-dimensional assessment systems may provide accurate information by assessing movements in all angles, natural movements may not be obtained since the 3D assessment systems could obtain data only in a limited space with limited movements. In addition, 3D systems are high in cost and require expert handlings (Lang et al., 2017). Recent studies have been conducted with inertia devices. These devices are comparatively inexpensive and aims to assess natural movements. These inertia devices, however, assess one-sided movements with an independent assessment device.
${ }^{*}$ Corresponding author: Haemi Jee (iD https://orcid.org/0000-0002-9066-1472 Department of Physical Therapy, Namseoul University, 91 Daehak-ro, Seobuk-gu, Cheonan 31020, Korea

E-mail: amyjee@nsu.ac.kr

Received: June 28, 2019 / Accepted: July 15, 2019
This is an Open Access article distributed under the terms of the Creative Commons Attribution Non-Commercial License (http://creativecommons.org/licenses/by-nc/4.0/) which permits unrestricted non-commercial use, distribution, and reproduction in any medium, provided the original work is properly cited. 
Human movements can be analyzed in various ways. Among various assessment methods, relative comparisons are commonly applied in human movements. Relative comparisons are made when two absolute values are compared to each other. In human kinematics, relative comparisons are made with one side to another. Usually, one side is assessed first and the counter side is assessed thereafter for comparisons. Although movements may be within the statistical means, each movement can produce different result for different comparative results.

In order to obtain more accurate comparative results, the ipsilateral and contralateral sides should be assessed at the same time for more accurate comparison. In order to assess movements of ipsilateral and contralateral sides simultaneously, a set of novice devices composed of gyroscopic and acceleration sensors was developed for synchronized assessment. This study analyzed synchronized movements of the abduction and adduction motions of lateral raises with two novice devices and the results were compared with results of the traditional gold-standard method.

\section{MATERIALS AND METHODS}

The study was conducted with 25 participants aged between 19 to 28 years with mean age of $22.1 \pm 2.34$ years. The study design was review and approved by the Inha University Ethics Committee. Prior to the study, the participants were thoroughly informed of the purpose and procedure of the experiment. The participants were agreed to participate in the study verbally and signed the informed content prior to participating in the study. The mean body mass index of the participants was $21.7 \pm 2.29 \mathrm{~kg} / \mathrm{m}^{2}$. As for the exercise habit of the participants, the subjects regularly performed exercise $2.3 \pm 2.23$ days per week and $34.3 \pm 36.68 \mathrm{~min}$ per day on the average at a low intensity of $2.7 \pm 2.80$ assessed by rate of perceived exertion (RPE) with scale 0 to 10 . All 25 participants were right handed with average dominant handedness score of $9.0 \pm 1.16$. The dominant handedness ranges from -10 to 10 with the left-handedness close to -10 and the right-handedness close to 10 , respectively.

\section{Experimental procedure}

The abduction and adduction motions were conducted by performing the lateral raises of the upper limbs. The procedures were performed according to a previous study (Noble et al., 1983). The RPEs with Borg scale of 0 to 10 were assessed immediately after each set of the lateral raises (Andersen et al., 2010; Noble et al., 1983). The participants were restrained from consuming a large- sized meal or drink prior to the test.

\section{Questionnaires}

Information on the general characteristics of the participants was obtained prior to conducting lateral raises. The questionnaires included information on the participants' physical and health status, exercise habit, and handedness. The Edinburgh Inventory was used to assess the handedness (Oldfield, 1971; Veale, 2014).

\section{Lateral raises}

Two dumbbells were used as free weights to be held by each hand of the participants. Low exercise intensity of $60 \%$ or less of one-repetition maximum was used during the lateral raises. The exercise intensity was calculated and obtained based on previously reported procedures (McBride et al., 2009; Morishita et al., 2018). The Borg scale ranging from 1 to 10 was used to assess the RPE prior to each bout. Before the initiation of the lateral raises, the participants were asked to be familiar with the dumbbells. The dumbbell weights ranged from 1 to $5 \mathrm{~kg}$. Following the completion of the questionnaires, the abduction and adduction motions were explained and shown to the participants. The participants were asked to conduct the lateral raises in controlled manner without a jerk or an accelerated movement and with each arm synchronized to each other. The participants were asked to stand with the feet-apart shoulder wide and hold each dumbbell on each hand slightly away from the body. The lateral raises were repeated twenty times or until fatigue.

\section{Novice motion sensors}

The hardware for the novice devices was the CC2650 SensorTag from Texas Instruments (Dallas, TX, USA) with accelerometer and gyroscope (Texas Instruments, 2019). The maximum measurement range of the linear acceleration from 3 -axis accelerometer and the angular velocity from 3 -axis gyroscope were $\pm 16 \mathrm{~g}$ and $\pm 2,000^{\circ} / \mathrm{sec}$, respectively. The hardware interrupt method on each sensor was utilized to synchronize the system time from the devices. The sensor node transmits the measured data to the data processing node via Bluetooth low energy communication. The transmitted raw data were processed to estimate the motion using the extended Kalman filter (Yun et al., 2006).

Two identical wireless devices that were synchronized were attached to both distal ends of the wrists. The kinematic data that includes synchronized position information of the angle $\left({ }^{\circ}\right)$, acceleration $\left({ }^{\circ} / \mathrm{sec}^{2}\right)$, and velocity $(\% / \mathrm{sec})$ were recorded every one-tenth of a second to a nearby smart-phone by real-time. Fully adducted 

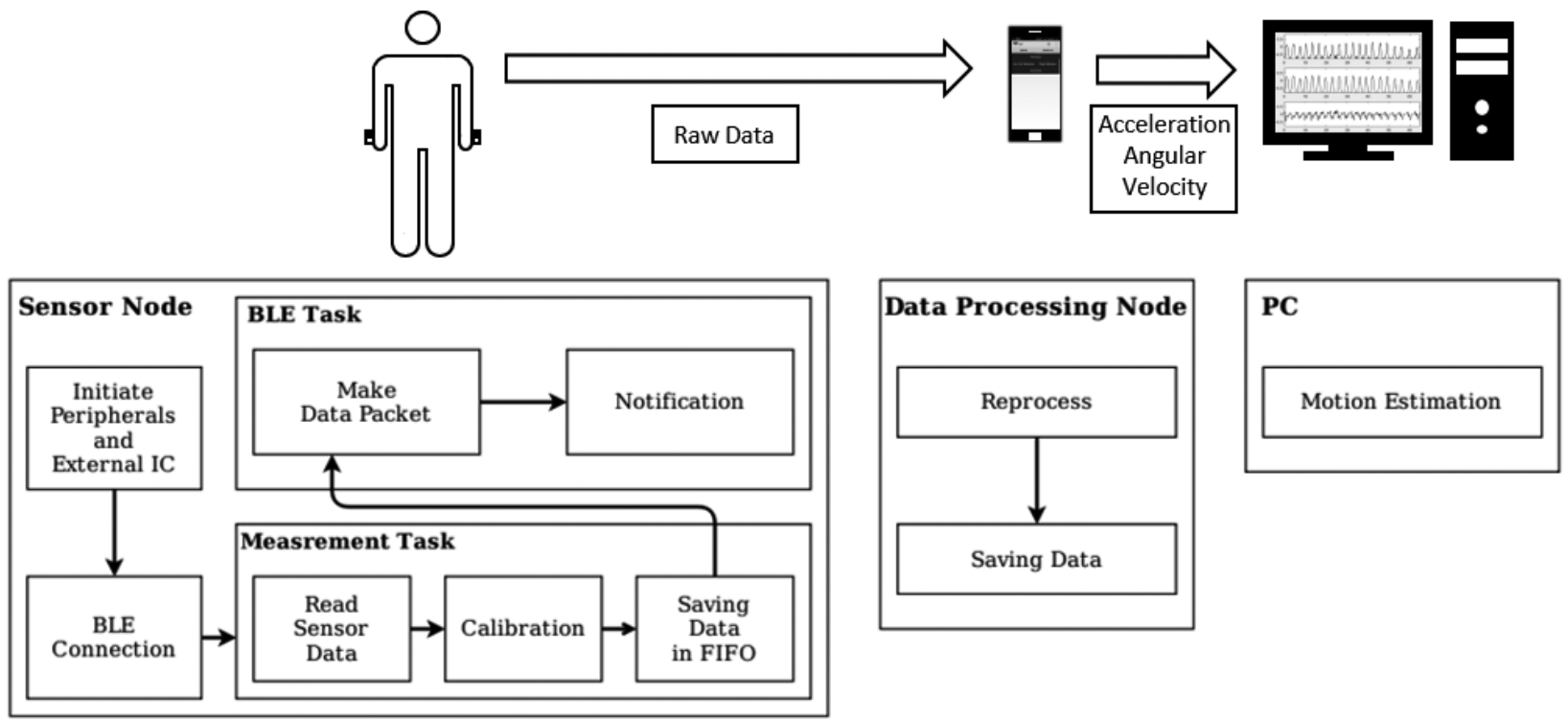

Fig. 1. Block diagram of the motion measurement system. BLE, Bluetooth low energy; IC, integrated circuit; FIFO, first-in-first-out.

and fully abducted angles, and corresponding acceleration and velocity were utilized for this study. The lateral raises were continuously conducted. The second adduction and adduction motions of the lateral raises were assessed for the analyses. The lateral raises were repeated after a sufficient washout period for test-retest comparisons (Fig. 1).

\section{Golden standard assessment}

A video camera was placed on a stool $1 \mathrm{~m}$ tall and $2 \mathrm{~m}$ away from the location designated for the lateral raises for full upper body shots. The motions were videoed as the participants stood facing the camera and after the initial practice motions. The videoed motions were captured by frame and analyzed according to previous studies (do Rosário, 2014; Macedo Ribeiro et al., 2017). The results from the captured video screens and devices were compared for golden standard comparisons.

\section{Statistical analysis}

The normality analysis by Shapiro-Wilks test was performed prior to the feasibility analysis $(P>0.05)$. All data sets were normally distributed with a very strong straight line of fit. The test-retest, and golden standard to the novice device result comparisons were conducted by using Pearson correlation and effect size (Cohen $d$ ) analyses. The correlation coefficient or agreement between 0.60 to 0.80 was considered 'substantial'; coefficients greater than 0.80 were regarded as 'excellent' (near perfect) (Jee et al., 2015; McGrath et al., 2006). The effect size which quantifies the difference between measurements was also calculated to further strengthen the degree of agreement between the results (McGrath et al., 2006). Effect size $\left(r^{2}\right)$ calculations were further conducted to observe the magnitude of difference between the two results. Effect sizes 0.2 to 0.3 were considered to have a 'small' effect, around 0.5 a 'medium' effect and 0.8 to infinity, a 'large' effect (Lininger and Riemann, 2016). Data were collected in a predesigned case data sheet and analyzed accordingly with MedCalc ver. 12.0 of MedCalc Software BVBA (Ostend, Mariakerke, Belgium). A $P$-value of 0.05 was used to determine the statistical significance.

\section{RESULTS}

The fully adducted angle or position and corresponding acceleration and velocity were compared. The feasibility assessment of the novice set of devices was first conducted through the test-retest comparisons. The overall mean angles assessed by the novice devices for the first full adduction and abduction positions were $8.6^{\circ} \pm 5.59^{\circ}$ and $92.8^{\circ} \pm 9.78^{\circ}$ and the second full adduction and abduction positions were $9.0^{\circ} \pm 5.62^{\circ}$ and $96.7^{\circ} \pm 14.10^{\circ}$, respectively. The overall mean angles assessed by the golden standard for the first full adduction and abduction positions were $9.0^{\circ} \pm 2.59^{\circ}$ and $96.6^{\circ} \pm 6.51^{\circ}$ and the second full adduction and abduction positions were $9.5^{\circ} \pm 2.91^{\circ}$ and $95.7^{\circ} \pm 2.91^{\circ}$, respectively. The overall mean acceleration values assessed by the novice devices for the first full adduction and abduction positions were $0.6 \pm 0.37 \%$ 
Table 1. Correlation coefficient and effect size calculations between the test-retest results of the novice devices and golden standard and novice devices results

\begin{tabular}{|c|c|c|c|c|}
\hline \multirow[b]{2}{*}{ Variable } & \multicolumn{2}{|c|}{ Correlation coefficient $r(P$-value) } & \multicolumn{2}{|c|}{ Effect size $\left(r^{2}\right)$} \\
\hline & $\begin{array}{l}\text { Novice test-retest } \\
\qquad(\mathrm{n}=25)\end{array}$ & $\begin{array}{l}\text { Novice vs. golden standard } \\
\qquad(\mathrm{n}=25)\end{array}$ & $\begin{array}{l}\text { Novice test-retest } \\
\qquad(\mathrm{n}=25)\end{array}$ & $\begin{array}{l}\text { Novice vs. golden standard } \\
\qquad(n=25)\end{array}$ \\
\hline \multicolumn{5}{|l|}{ Angle } \\
\hline Range (min-max) & $0.76-0.88(<0.01 *)$ & $0.83-0.81(<0.01 *)$ & $0.34-0.09$ & $0.42-0.29$ \\
\hline \multicolumn{5}{|l|}{ Acceleration } \\
\hline Range (min-max) & $0.84-0.86\left(<0.01^{*}\right)$ & - & $0.02-0.28$ & - \\
\hline Mean & 0.84 & 0.82 & 0.18 & 0.36 \\
\hline
\end{tabular}

Min, full adduction position; Max, full abduction position; Acceleration, instantaneous acceleration.

${ }^{*} P<0.05$.

Table 2. Correlation coefficient and effect size calculations between the difference in left and right upper limb test-retest results of the novice devices and golden standard and novice devices results

\begin{tabular}{|c|c|c|c|c|}
\hline \multirow{2}{*}{ Variable } & \multicolumn{2}{|c|}{ Correlation coefficient $r(P)$} & \multicolumn{2}{|c|}{ Effect size $\left(r^{2}\right)$} \\
\hline & Novice test-retest & Novice vs. golden standard & Test-retest & Novice vs. golden standard \\
\hline \multicolumn{5}{|l|}{ Angle difference } \\
\hline Range (min-max) & $0.66-0.70\left(<0.01^{*}\right)$ & $0.73\left(0.01^{*}\right)-0.78\left(0.02^{*}\right)$ & $0.30-0.23$ & $0.43-0.37$ \\
\hline \multicolumn{5}{|c|}{ Acceleration difference } \\
\hline Range (min-max) & $0.69-0.65\left(<0.01^{*}\right)$ & - & $0.19-0.24$ & - \\
\hline Mean & 0.68 & 0.76 & 0.26 & 0.40 \\
\hline
\end{tabular}

Min, left-right full adduction results; Max, left-right full abduction results.

${ }^{*} P<0.05$.

$\sec ^{2}$ and $3.1 \pm 1.18^{\circ} / \mathrm{sec}^{2}$ and the second full adduction and abduction positions were $1.0 \pm 0.58^{\circ} / \mathrm{sec}^{2}$ and $2.5 \pm 1.07 \% \mathrm{sec}^{2}$, respectively.

In addition, differences in the results of the left and right upper arms were compared. The mean angle differences assessed by the novice devices for the first full adduction and abduction positions were $-2.1^{\circ} \pm 5.02^{\circ}$ and $1.1^{\circ} \pm 9.15^{\circ}$ and the second full adduction and abduction positions were $-2.6^{\circ} \pm 4.66^{\circ}$ and $1.3^{\circ} \pm 8.52^{\circ}$, respectively. The mean angle differences assessed by the golden standard for the first full adduction and abduction positions were $-0.1^{\circ} \pm$ $1.75^{\circ}$ and $0.4^{\circ} \pm 5.68^{\circ}$ and the second full adduction and abduction positions were $-0.5^{\circ} \pm 1.53^{\circ}$ and $-0.2^{\circ} \pm 4.78^{\circ}$, respectively. The mean acceleration differences assessed by the novice devices for the first full adduction and abduction positions were $-0.5 \pm$ $0.62^{\circ} / \mathrm{sec}^{2}$ and $0.6 \pm 1.28^{\circ} / \mathrm{sec}^{2}$ and the second full adduction and abduction positions were $-0.4 \pm 0.43 \% \mathrm{sec}^{2}$ and $0.6 \pm 1.29^{\circ} / \mathrm{sec}^{2}$, respectively. Instantaneous accelerations could not be obtained from the traditional golden standard method. Significant differences between the results were not observed for all comparisons.

Pearson correlation and effect size analyses were conducted between the test and retest values of the novice devices and between the novice device and golden standards results as in Table 1. Excellent to substantial correlating relationships were observed be- tween the test-retest values and test and golden standard values with overall small effect sizes. Pearson correlation and effect size analyses were further conducted between the differences in the left and right upper limb position and acceleration values as in Table 2. Substantial correlating relationships were observed with small effect sizes between the test-retest values and test and golden standard values.

\section{DISCUSSION}

Evaluating human motion is a difficult task especially for the upper limbs since the movement involves complex 3D motions (Bonnechère et al., 2018). Comparative assessments become more complex when the motion analysis involves two limb comparisons. The recent technological advancements allowed possible application of miniaturized assessment devices for natural movement assessment (Iosa et al., 2016; Picerno, 2017). Comparison of bilateral motions is necessary for relative comparison between the ipsilateral and contralateral sides such as left and right upper limbs. A pair of wrist-worn novice devices were developed to assess motions of two different limbs at the same time for two limb motion comparisons. The novice devices were developed for real-time comparisons of the asymmetrical motion of the upper limbs for early de- 
tection of possible misalignment of either limb.

The lateral raise motions were compared between novice device measured test-retest results and golden standard measurement results. Excellent Pearson correlating coefficient values $(r \geq 0.76, P=$ $0.01)$ and small effect sizes $\left(r^{2} \leq 0.43\right)$ showed good correlation between the test-retest results of the left and right upper limbs. Moreover, the data comparison between the novice device and the golden standard results also showed excellent correlation $(r \leq 0.81$, $P=0.01)$ and small effect sizes $\left(r^{2} \leq 0.21\right)$ results of the left and right upper limbs. The correlating values for the differences in angles and accelerations were substantial $\left(r^{2} \geq 0.65\right)$. However, considering comparatively low correlation coefficient value between the left and right limbs of $0.65(P<0.01)$ in this study, the testretest correlating values were reliable.

In previous studies assessing the correlation between two devices, the correlating values ranged from 0.59 to $0.78(P<0.05)$ (Bailey et al., 2014; Lang et al., 2017; Lee et al., 2019). Considering that previous studies measured only the activity levels of the upper limbs by accelerometer-based devices, the results of this study are accurate in measuring precise location and kinematics. Other studies which observed validity of devices for kinematics of the upper limbs showed high correlating values of 0.95 or above (Cutti et al., 2008; El-Gohary and McNames, 2012). These studies compared the fixed motions instead naturally occurring motions. This study compared two different test results for correlation analysis for the test-retest assessment. Although the same instructions were given, manually performed movements may provide different results every time. Correlation coefficients of the left and right motions were between 0.61 and $0.65(P<0.01)$. Moreover, correlation coefficient of two different trails was 0.85 $(P=0.02)$ in this study. Such results indicate that correlation values may different due to different kinematic results of the human motions.

The novice devices have been proven for their feasible for comparing simultaneous lateral raise motions of the left and right upper limbs in this study. In addition, the wrist-attachable novice devices have the advantage of being portable and inexpensive. It could be further designed to be fixed in various joints for different kinematic motion analysis. In addition, the paired devices could readily identify dyskinetic motions readily. The assessed results could be used to evaluate various upper limb motions to provide for both functional and clinical purposes by assessing natural movement in real-world settings.

Although practical in performing various functions of the upper limbs, the possibility for musculoskeletal disorder is also com- mon. Early and accurate detection of dyskinesia is vital for proper prevention and rehabilitation. The wrist-worn motion sensor devices have been gaining significant attention from researchers and clinician for their practical application in everyday situation and practical cost (Hayward et al., 2016). Wrist-work devices have been majorly utilized for intensity, duration, and ratio of use of the limbs (Lee et al., 2019). Since more than $50 \%$ of the upper limb activities involve bilateral movements, assessment of bilateral kinematics is a must for understanding the upper limbs (Lee et al., 2019).

There were some limitations to the study. One of the limitations was that the comparison was done in one-dimensional plane. Although the novice device could obtain kinematic information in $6^{\circ}$ of motion (forward/back, up/down, left/right, yaw, pitch, and roll), the study only utilized motion in frontal plane. Since the video camera could only be handled from the frontal plane, the results could only be compared for one dimension. Further studies should be conducted for the movements in three anatomical planes of frontal, sagittal, and transverse planes. Another limitation of this study is that the kinematic movement was assessed only by lateral raises with abduction and adduction motions. One of the major purposes of this study was to develop and assess a novice device that could assess everyday natural kinematic motions. Although one type of kinematic motion was evaluated for feasibility, application of other movements could be easily conducted with adjustment of the software program that was developed for the novice device. In addition, categorizing purposeful upper limb motion can provide meaningful information for clinical settings (Hayward et al., 2016; Lee et al., 2019). Homogenous natural of the participants and the sample size would be another limitation of the study. This study was done with healthy participants with age ranges of twenty to thirty. Observation with other heterogeneous groups of subjects should be observed for diverse application of the novice devices.

The novice set of devices is feasible for use in comparison of the motions of two upper-limb motions and has the great advantage of being portable and low in cost. These set of novice device could be vitally utilized for assessing asymmetry in kinematic motion of the upper limbs for early rehabilitation and proper exercise intervention for prevention of misaligned motion during exercise.

\section{CONFLICT OF INTEREST}

No potential conflict of interest relevant to this article was reported. 


\section{ACKNOWLEDGMENTS}

Funding for this paper was provided by Namseoul University year 2019 .

\section{REFERENCES}

Andersen LL, Andersen CH, Mortensen OS, Poulsen OM, Bjørnlund IB, Zebis MK. Muscle activation and perceived loading during rehabilitation exercises: comparison of dumbbells and elastic resistance. Phys Ther 2010;90:538-549.

Bailey RR, Klaesner JW, Lang CE. An accelerometry-based methodology for assessment of real-world bilateral upper extremity activity. PLoS One 2014;9:e103135.

Bonnechère B, Sholukha V, Omelina L, Van Sint Jan S, Jansen B. 3D analysis of upper limbs motion during rehabilitation exercises using the Kinect $^{\mathrm{TM}}$ sensor: development, laboratory validation and clinical application. Sensors (Basel) 2018;18.

Cutti AG, Giovanardi A, Rocchi L, Davalli A, Sacchetti R. Ambulatory measurement of shoulder and elbow kinematics through inertial and magnetic sensors. Med Biol Eng Comput 2008;46:169-178.

do Rosário JL. Photographic analysis of human posture: a literature review. J Bodyw Mov Ther 2014;18:56-61.

El-Gohary M, McNames J. Shoulder and elbow joint angle tracking with inertial sensors. IEEE Trans Biomed Eng 2012;59:2635-2641.

Hadzic V, Sattler T, Veselko M, Markovic G, Dervisevic E. Strength asymmetry of the shoulders in elite volleyball players. J Athl Train 2014;49: 338-344.

Hayward KS, Eng JJ, Boyd LA, Lakhani B, Bernhardt J, Lang CE. Exploring the role of accelerometers in the measurement of real world upperlimb use after stroke. Brain Impairment 2016;17:16-33.

Iosa M, Picerno P, Paolucci S, Morone G. Wearable inertial sensors for human movement analysis. Expert Rev Med Devices 2016;13:641-659.

Jee H, Kim J, Kim C, Kim T, Park J. Feasibility of a semi-computerized line bisection test for unilateral visual neglect assessment. Appl Clin Inform 2015;6:400-417.

Lang CE, Waddell KJ, Klaesner JW, Bland MD. A method for quantifying upper limb performance in daily life using accelerometers. J Vis Exp 2017;122:e55673.

Lee SI, Liu X, Rajan S, Ramasarma N, Choe EK, Bonato P. A novel upperlimb function measure derived from finger-worn sensor data collected in a free-living setting. PLoS One 2019;14:e0212484.
Lininger M, Riemann BL. Statistical primer for athletic trainers: using confidence intervals and effect sizes to evaluate clinical meaningfulness. J Athl Train 2016;51:1045-1048.

Macedo Ribeiro AF, Bergmann A, Lemos T, Pacheco AG, Mello Russo M, Santos de Oliveira LA, de Carvalho Rodrigues E. Reference values for human posture measurements based on computerized photogrammetry: a systematic review. J Manipulative Physiol Ther 2017;40:156168.

Maly T, Zahalka F, Mala L, Cech P. The bilateral strength and power asymmetries in untrained boys. Open Med (Wars) 2015;10:224-232.

Matsuki K, Matsuki KO, Mu S, Yamaguchi S, Ochiai N, Sasho T, Sugaya $\mathrm{H}$, Toyone T, Wada Y, Takahashi K, Banks SA. In vivo 3-dimensional analysis of scapular kinematics: comparison of dominant and nondominant shoulders. J Shoulder Elbow Surg 2011;20:659-665.

McBride JM, McCaulley GO, Cormie P, Nuzzo JL, Cavill MJ, Triplett NT. Comparison of methods to quantify volume during resistance exercise. J Strength Cond Res 2009;23:106-110.

McGrath RE, Meyer GJ. When effect sizes disagree: the case of $r$ and $d$. Psychol Methods 2006;11:386-401.

Morishita S, Tsubaki A, Takabayashi T, Fu JB. Relationship between the rating of perceived exertion scale and the load intensity of resistance training. Strength Cond J 2018;40:94-109.

Noble BJ, Borg GA, Jacobs I, Ceci R, Kaiser P. A category-ratio perceived exertion scale: relationship to blood and muscle lactates and heart rate. Med Sci Sports Exerc 1983;15:523-528.

Oldfield RC. The assessment and analysis of handedness: the Edinburgh inventory. Neuropsychologia 1971;9:97-113.

Picerno P. 25 years of lower limb joint kinematics by using inertial and magnetic sensors: A review of methodological approaches. Gait Posture 2017;51:239-246.

Rettig O, Krautwurst B, Maier MW, Wolf SI. Definition of anatomical zero positions for assessing shoulder pose with 3D motion capture during bilateral abduction of the arms. BMC Musculoskelet Disord 2015;16: 383.

Texas Instruments. Embedded development hardware kits \& boards [Internet]. Dallas (TX): Texas Instruments; c2019 [cited 2019 Jun 20]. Available from: http://www.ti.com/tools-software/sensortag.html.

Veale JF. Edinburgh Handedness Inventory - Short Form: a revised version based on confirmatory factor analysis. Laterality 2014;19:164-177.

Yun X, Bachmann ER. Design, Implementation, and experimental results of a quaternion-based kalman filter for human body motion tracking. IEEE Trans Robot 2006;22:1216-1227. 